\title{
Actividades físico recreativas para mejorar la memoria en el adulto mayor (análisis descriptivo)
}

DOI: https://doi.org/10.33262/ap.v2i4.12

\begin{abstract}
(c) (1) (9)(2)
Physical recreational activities to improve memory in the elderly adult (descriptive analysis).
\end{abstract}

Mónica Cabezas Cabezas Flores., ${ }^{1}$ Alex Stalin Otañez Reyes., ${ }^{2}$ Jefferson Gerardo Tipán Coyago. ${ }^{3} \&$ Ruth Sayonara Rios Bayas. ${ }^{4}$

\begin{abstract}
.
This research work is a valuable resource for those who fulfill the great task of working with the elderly input, analysis of recreational physical activity is sustained and this as involved in improving memory in older people, seeking always to contribute in the prevention and treatment of diseases considered non-communicable, the goal is to help adults maintain a good quality of life, a non-experimental descriptive study which revealed research findings that older adults have problems made with short-term memory and preserve more long-term memory, so with stimulation of cognitive memory is to delay the proceedings of memory impairment and prevents memories and valuable information are cleared for people. Finally conclusions and recommendations are made in line with the research.
\end{abstract}

Keywords: Elderly, Recreational Activities, Memory.

\section{Resumen.}

\footnotetext{
${ }^{1}$ Universidad de las Fuerzas Armadas “ESPE”. Quito, Ecuador. mmcabezas@espe.edu.ec

${ }^{2}$ Universidad de las Fuerzas Armadas "ESPE”. Quito, Ecuador. asotanez@espe.edu.ec

${ }^{3}$ Universidad de las Fuerzas Armadas "ESPE”. Quito, Ecuador. jgtipan@espe.edu.ec

${ }^{4}$ Universidad de las Fuerzas Armadas “ESPE”. Quito, Ecuador. rsrios@espe.edu.ec
} 
El presente trabajo investigativo constituye un aporte valioso para quienes cumplen la gran tarea de trabajar con las personas adultas mayores, se sustenta el análisis de las actividades físico recreativas y esto como interviene en el mejoramiento de la memoria en el adulto mayor, que buscan siempre contribuir en la prevención y tratamiento de las enfermedades consideradas como no trasmisibles, el objetivo es ayudar a que las personas adultas mantengan una buena calidad de vida, se realizó un estudio descriptivo no experimental el cual arrojo como resultados de la investigación que los adultos mayores presentan problemas con la memoria a corto plazo y preservan más la memoria a largo plazo, por lo que con la estimulación de la memoria cognitiva se va a retrasar el curso del proceso de deterioro de la memoria y se evita que se borren recuerdos e información valiosa para las personas. Por último se elaboran las conclusiones y recomendaciones en consonancia con la investigación.

Palabras claves: Adulto Mayor, Actividades Recreativas, Memoria.

\section{Introducción.}

En la actualidad se vive más años que en otras épocas, como resultado de las mejoras ocurridas en la higiene, por la práctica de ejercicios físicos y programas recreativos, por los cambios de hábitos, en especial los vinculados con una alimentación saludable y equilibrada en función de las actividades diarias del adulto mayor, así como los avances de las ciencias médicas para la prevención y asistencia oportuna en enfermedades y lesiones propias de esta etapa de la vida, provocando una mejor calidad de vida y estado de bienestar.

La práctica regular de actividades físicas y recreativas, plantea un doble reto: la oportunidad de aprovechar al máximo unos años más de vida y una mayor necesidad de hacer frente y en mejores condiciones a las incapacidades que surgen en la tercera edad, propiciando vivir más años, no agregando días a los años, sino placer y confort hasta donde más se pueda.

Cabe señalar que las actividades física y recreativas son de vital importancia para las personas de la tercera edad, ya que no sólo se dirigen a mejorar los segmentos contráctiles del organismo facilitando coordinación, estabilidad, agilidad, potencia muscular, reacciones defensivas ante estímulos externos o a desempeñar un momento lúdico, sino que además está orientada constructivamente a lograr la participación, la reinserción social del individuo que es fundamental, pues el interés del adulto mayor debe dirigirse a la unidad grupal.

El propósito de esta investigación es analizar las ventajas que tienen las actividades físicas y recreativos para los adultos, como alternativa para mejorar la calidad de vida, rehabilitando y previniendo enfermedades de múltiples orígenes.

El envejecimiento natural como causa implica disminución de la capacidad funcional del ser humano, en donde el deterioro biológico se pone de manifiesto de forma general tanto en lo físico como en lo mental. Este proceso en la actualidad representa un reto para las 
diferentes instituciones médicas y los jefes de estado que tienen a su cargo el cuidado de la salud del adulto mayor.

Los adultos mayores pueden llevar a cabo diferentes actividades diarias y realizar aquellas que aseguren el mantenimiento personal (comer, bañarse, vestirse, entre otras.) y que se consideran un ingrediente esencial de la vida; sin embargo, también pueden involucrarse con otros en algo más que las actividades básicas, tomar parte en el medio ambiente externo $\mathrm{y}$ en actividades que son enriquecedoras.

La vida diaria está sujeta a un ritmo en el cual es casi inevitable la sobre tensión o estrés en cualquier etapa, aparejado a esto el sedentarismo se hace acompañar de efectos negativos que pueden acarrear enfermedades o lesiones que, con el transcurso del tiempo, podrían traer consigo una secuela de padecimientos o males (como diabetes e hipertensión, entre otras), al no ser atendidos a tiempo.

Es así como una de las primeras manifestaciones de enfermedades en el adulto mayor se produce a través de alteraciones de la funcionalidad. Esta constituye el signo de alerta más importante. De aquí la importancia fundamental de la medición permanente de la funcionalidad en el adulto mayor

\section{Marco teórico conceptual.}

\section{¿Qué es actividades físicas recreativas?}

Es una disposición particular positiva y favorable de cambio y regeneración, (...) Bajo este nuevo concepto se desarrolla la recreación dirigida que debe cumplir la condición de volver a crear, divirtiendo mediante una actitud activa y una implicación en grupo (Camerino, 2000)

Para poder definir una actividad como recreativa, debe cumplir al menos tres requisitos: libertad de elección y realización, motivación personal para su desarrollo y ejecución placentera para la persona que la realiza (Trillas, 1990)

La actividad física recreativa debería ser un juego en el que también se ha creado un marco de fantasía. Sin embargo, es evidente que en muchas ocasiones no lo es. Falta ese elemento lúdico, olvidando a menudo por el propio afán de competitivo que antepone el deseo de victoria a la propia recreación

Es evidente que dentro de los dos grandes discursos que se plantea en la actividad física que define El VI congreso mundial de ocio:

a) El rendimiento, que implica la búsqueda sistematizada de un producto final, selección, la competición y se basa en la concepción del cuerpo como máquina. Se enmarca en una experiencia productiva, profesional y elitista y está fuertemente 
sustentada política, mediática y económicamente, por otra parte.

b) La participación: que consiste en incrementar la participación en la cultura del movimiento. Se estructura en torno a la inclusión, igualdad, disfrute, proceso, satisfacción, vivencia, libertad.

Las actividades físicas recreativas toman partido por esta segunda opción, la única que a lo largo de la historia ha permitido crear ese marco lúdico que conlleva el juego y la recreación cuya práctica debe:

- Deparar bienestar

- Facilitar la comunicación interpersonal

- Desarrollar la creatividad

- Expandir horizontes personales

- Potencia la recreación personal y aumenta la autoestima

- Responde a necesidades individuales

- Reforzar el deseo de practicar esa actividad

\section{¿Quiénes son las personas adultas mayores?}

Debido al aumento de la esperanza de vida y a la disminución de la tasa de fecundidad, la proporción de personas mayores de 60 años está aumentando más rápidamente que cualquier otro grupo de edad en casi todos los países. El envejecimiento de la población puede considerarse un éxito de las políticas de salud pública y el desarrollo socioeconómico, pero también constituye un reto para la sociedad, que debe adaptarse a ello para mejorar al máximo la salud y la capacidad funcional de las personas mayores, así como su participación social y su seguridad.

\section{¿Por qué es importante la actividad física para las personas adultas mayores?}

"Los beneficios de la actividad física están bien documentados. Existe evidencia de que una vida sedentaria es uno de los riesgos de salud modificables más altos para muchas condiciones crónicas que afectan a las personas adultas mayores, tales como la hipertensión, las enfermedades del corazón, el accidente cerebro vascular, la diabetes, el cáncer y la artritis. Aumentar la actividad física después de los 60 años tiene un impacto positivo notable sobre estas condiciones y sobre el bienestar general.” (Washington, 2002) 


\section{Trastornos que pueden interferir con el funcionamiento de la memoria}

El adulto mayor con un posible trastorno de la memoria, debe ser sometido a un estudio clínico, con una evaluación neurológica que incluya el examen de las funciones cognitivas que puede ser realizado por cualquier médico e incluso por personal paramédico. Si se requiere de un examen más detallado, puede ser necesaria la cooperación de una neuropsicología, así como la realización de pruebas más complejas.

Para que una información sea registrada y evocada adecuadamente, es decir, para que funcione la memoria, es necesario que otras funciones neuropsicológicas estén intactas. Así, las fallas en atención, lenguaje, comunicación y motivaciones, pueden dificultar el funcionamiento de la memoria sin que esta función en sí misma esté afectada.

\section{Trastornos de la memoria:}

- Depresión

- Trastornos de la atención

- Afasia

- Defectos sensoriales (visuales, auditivos)

\section{Importancia de la práctica sistemática de actividad física}

La práctica sistemática de actividades físicas en cualquier edad permite ante todo:

- Desarrollar procesos, cualidades y funciones psicológicas necesarias para alcanzar el éxito en las propias acciones motrices y en otras actividades cotidianas.

- Profundizar la convicción de que somos capaces de controlar con efectividad nuestro propio cuerpo.

- Aumentar nuestras vivencias de éxito, lo que favorece el desarrollo de la autoconfianza y la autovaloración.

- Disfrutar de la práctica de actividades que provocan una gran saturación emocional y satisfacción personal.

- Sentirnos aptos para alcanzar resultados concretos, en unión de otros, lo cual profundiza la identificación a determinado grupo.

- Vivenciar positivamente la capacidad de mantenernos activos. 


\section{Efectos beneficiosos de la actividad física a nivel psicológico:}

- Enseña a superarse.

- Eleva la calidad de vida.

- Es anti-estrés.

- Ayuda a liberar impulsos agresivos.

- Ayuda a controlar la ansiedad.

- Mantiene alta la motivación.

- Mantiene alta la auto-estima.

- Colabora en el desarrollo afectivo, social, intelectual y motor.

- Puede aliviar estados de ánimo adversos.

- Busca mantener el aspecto físico que incrementa el optimismo y el auto concepto.

- A nivel mental produce un efecto distractor y relajante de la cotidianeidad.

- Alivia tensiones corporales.

- Equilibra el estado emocional.

- Efectos beneficiosos de la actividad física a nivel fisiológico:

\section{Efectos inmediatos:}

- Regulación del nivel de glucosa;

- Regulación de algunos neurotransmisores como la catecolamina;

- Estimula la noradrenalina y la adrenalina;

- Potencia la elaboración de endorfinas;

- Mejora el sueño.

\section{Efectos a largo plazo:}

- Sensación de bien estar físico;

- Fortalecimiento de los sistemas cardiovascular y respiratorio, 
incrementando a la cantidad de oxígeno que llega a los diferentes órganos y tejidos corporales.

- Incremento de la resistencia muscular, con los consiguientes efectos benéficos sobre la autonomía personal.

\section{Objetivo del estudio.}

Sustentar un análisis sobre las actividades físico recreativo para mejorar la memoria en el adulto mayor.

\section{Métodos.}

Esta investigación se clasifica de tipo descriptivo no experimental, por solo constatar cómo es él es estado del funcionamiento cognitivo del adulto mayor y mediante la intervención de actividades físico recreativas mejorar la memoria en el adulto mayor.

\section{Recomendaciones}

- Realizar actividad física de manera frecuente para contribuir a la mejora de la memoria y la capacidad cognoscitiva.

- Recopilar diariamente las actividades realizadas en un cuaderno a manera de diario.

- Mantener una alimentación balanceada para un óptimo funcionamiento tanto físico como mental.

- Ejecutar los ejercicios de forma progresiva y teniendo en cuenta que todo cambio se va dando de manera progresiva.

\section{Conclusiones.}

- La teoría abordada en la investigación, sirvió de sustento para cumplir con el objetivo previsto.

- Como resultado del estudio realizado se pudo constatar que los adultos mayores presentan problemas con la memoria a corto plazo y preservan más la memoria a largo plazo, coincidiendo con lo que aparece en la bibliografía consultada.

\section{Referencias bibliográficas.}

Camerino. (2000). Aportaciones del deporte recreativo.

Trillas. Martínez Gámez, 1. (1990). Actividades físico-recreativas.

Washington. (2002). Promover un estilo de vida para las personas Adultas Mayores. 
Ramírez, R. (2012). El sedentarismo es un factor predictor de hipertrigliceridemia, obesidad central y sobrepeso. Pesquisa en base de dados.

Rosa, S. M. (1er trimestre 2006). Sedentarismo y salud: efectos beneficiosos de la actividad física. American College of Sports Medicine.

Sarmiento, D. O. (2012). Colombia, país sedentario. El Espectador.

http://www.efdeportes.com/efd149/la-ejercitacion-de-la-memoria-del-adulto- mayor.htm http://bvs.sld.cu/revistas/mfr/vol_4_2_12/mfr06212.htm

http://www.efdeportes.com/efd150/la-actividad-fisica-recreativa-para-la- comunidad.htm

file://C:/Users/CHINO/Downloads/Dialnet-

LasActividadesFisicorecreativasInstrumentosDeInter-2547380.pdf

http://catarina.udlap.mx/u_dl_a/tales/documentos/lar/dionne_e_mf/capitulo1.pdf

http://weblog.maimonides.edu/gerontologia2007/2007/12/los_beneficios_de_la_re creacio.html

http://www.efdeportes.com/efd181/actividades-fisicas-para-la-vida-diaria.htm

http://www.efdeportes.com/efd152/actividades-fisicas-con-caracter-ludico-para-el- adultomayor.htm.

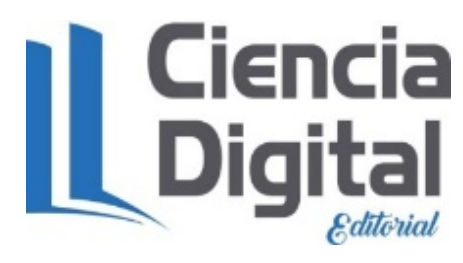




\section{Para citar el artículo indexado}

Cabezas Flores, M. C., Otañez Reyes, A. S., Tipán Coyago, J. G., \& Rios Bayas, R. S. (2020). Actividades físico recreativas para mejorar la memoria en el adulto mayor (análisis descriptivo). AlfaPublicaciones, 2(4), 51-59. https://doi.org/10.33262/ap.v2i4.12

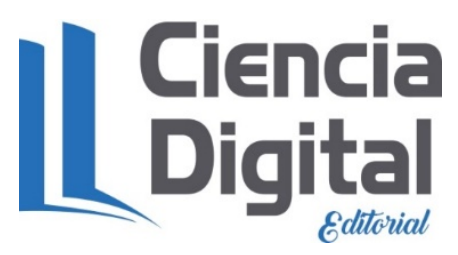

El artículo que se publica es de exclusiva responsabilidad de los autores y no necesariamente reflejan el pensamiento de la Revista Alpha Publicaciones.

El artículo queda en propiedad de la revista y, por tanto, su publicación parcial y/o total en otro medio tiene que ser autorizado por el director de la Revista Alpha Publicaciones.
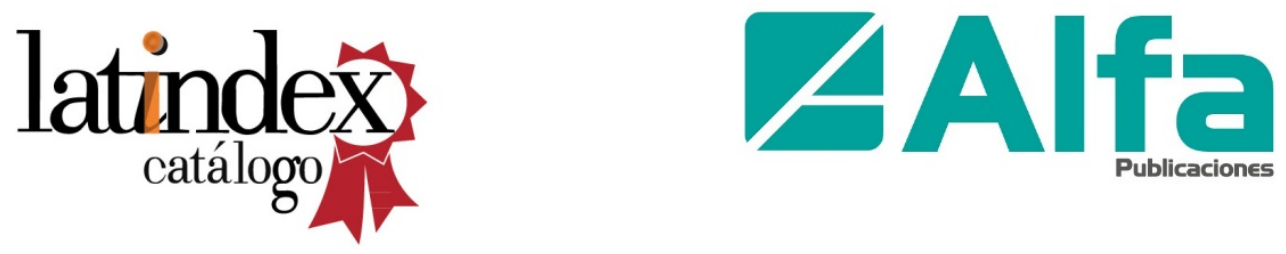